\title{
Preparation and release kinetics of betulinic acid/ CS drug-loaded microspheres
}

\author{
Hao Ran Zhou ${ }^{1}$, Xiao Jiao FANG ${ }^{2}$, Jin LV' , De Xin WANG ${ }^{2}$ \\ ${ }^{1}$ The University Key Laboratory of Fine Chemical Industry of Heilongjiang Province ,Chemistry and Chemical Engineering, Qiqihar University \\ 161006, China \\ ${ }^{2}$ School of Material Science and Engineering, Harbin University of Science and Technology,Harbin 150080, China
}

\begin{abstract}
Chitosan(CS) is the unique alkaline polysaccharide in nature, because of its perfect biocompatibility and degradability, it is widely used in medicine, soft release and control release. The betulinic acid is the derivative of betulin, it has many pharmacological activities, such as anti- inflammatory, antitumor, anti-malaria and anti-HIV. In this paper, the Span- 80 was used as emulsifiers, the glutaraldehyde was used as crosslinker. With the method of orthogonal experiment, the preparation technology was optimized. The microspheres were characterized by SEM and its degree of crosslinking, drug-loading rate and encapsulation efficiency were tested at the same time.
\end{abstract}

\section{Introduction}

Chitosan(CS) is the unique alkaline polysaccharide in nature, because of its perfect biocompatibility and degradability, it is widely used in medicine, soft release and control release. When the CS is used for drug carrier, it can prolong the application time of the medicine, reduce the adverse effect, increase the stability of the medicine and increase the permeability of cell membrane, it is a widely studied pharmaceutical excipient. The betulinic acid is the derivative of betulin, at first, it is extracted from birch bark by physical methods, then, with the development of the study, many other chemical methods were found to synthesis the betulinic acid at a high rate. As a result, the betulin acid has become a widely source. It not only has many pharmacological activities, such as anti- inflammatory, antitumor, anti-malaria and anti-HIV, but also low toxicity and with high safety index, so it is widely studied in the area of antitumor and anti-HIV.

Because the microspheres particle size homogeneous degree will directly affect the loading of betulinic acid/CS microspheres, so we have to achieve a relatively uniform levels of the bland microspheres of CS. In this paper, the Span- 80 and Twen- 80 were used as emulsifiers, the formaldehyde and glutaraldehyde were used as crosslinker. With the method of orthogonal experiment, the preparation technology of betulinic acid / CS microspheres was optimized. Above all, we prepared the microspheres of CS with uniform particle size, then we prepared the microspheres of betulinic acid and CS. We characterized the microspheres with the use of FT-IR, SEM and the test of the degree of crosslinking, we tested the drug-loading rate and encapsulation efficiency at the same time.
We studied the factors which will have an influence on the microspheres, such as the dosage of emulsifier and crosslinker, the dosing ratio of betulinic acid and CS. Then, we determined the optimum technological conditions: the molecular weight

\section{Preparation of the betulinic acid/CS microspheres}

The CS was was added in a round flask and dissolved in acetic acid (the concentration is $2 \%$ ), then put the flask in water bath and stirred until it mixed evenly, the betulinic acid was dissolved in chloroform. At the same time, the emulsification system was prepared: A certain amount of atolein and Span- 80 were mixed in a round flask, the mixture was stirred evenly in water bath, too.

Emulsion reaction: $30 \mathrm{~mL}$ of the CS-acetic acid mixture was taken out and added in the emulsification system with use of injector to control its flow rate, the temperature was $50^{\circ}$ and the process lasted for about $30 \mathrm{~min}$.

Cross-linking reaction: After the emulsion reaction, a certain amount of glutaraldehyde was added, then the cross-linking started, keep the temperature of $50^{\circ} \mathrm{C}$ and it lasted for about $3 \mathrm{~h}$.

After cross-linking process, the mixture was poured out, laid up until the solution was layered, then poured out the supernatant, a certain amount of petroleum ether was added, stirred and laid up, then poured out the supernatant, repeated the process for two times.

Washed the mixture with a certain amount of isopropanol and vacuum filtration at the same time, the solid we obtained is the betulinic acid/CS microspheres. 
The microspheres were laid on a clean watch glass and put it in an oven at the temperature of $50^{\circ} \mathrm{C}$.

\section{Test of the betulinic acid/CS microspheres Analysis of SEM}

The relatively uniform particle size of betulinic acid/CS microspheres is quite important for drug-loading, encapsulation efficiency and slow-release effect. As a result, the best process condition was determined by orthogonal test. The SEM was used to test the change caused by modification, the result is as following:

(a) the SEM image before modification

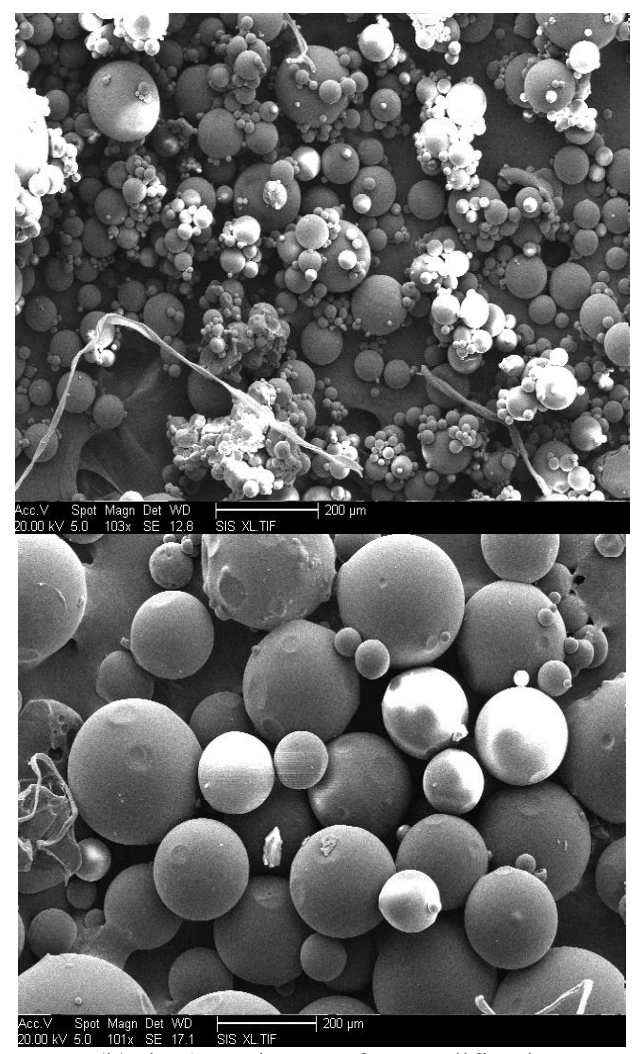

(b) the SEM image after modification

Fig.1 SEM images of the betulinic acid/CS microspheres

It can be concluded that the degree of particle size uniformity has been improved obviously, the particle size of (a) is ranged from 30 to $180 \mu \mathrm{m}$, the particle sized distribution is so wide, but the uniformity of particle size after modification is improved, primarily fasten on $100-150 \mu \mathrm{m}$.

\section{Analysis of the degree of crosslinking}

To determine the crosslinking degree of betulinic acid/CS microspheres[1-3], the drug was extracted with the use of extractor, and the solution used in this process is glacial acetic acid, its concentration is $0.5 \%$, the extraction last for about $24 \mathrm{~h}$. The weight of the microspheres should be weighed before and after the extraction, the product was put in an oven and weighed it after dried. The degree of crossslinking could be calculated and the result is shown as Tab. 1:

Tab. 1 Effect of glutaraldehyde dosage on the crosslinked degree of microsphere

\begin{tabular}{|c|c|c|c|c|}
\hline \multirow{2}{*}{$\begin{array}{c}\text { Dosage of } \\
\text { glutaraldeh } \\
\text { yde }(\mathrm{mL})\end{array}$} & \multicolumn{2}{|c|}{$\begin{array}{l}\text { Mass before } \\
\text { extraction(g) }\end{array}$} & \multirow{2}{*}{$\begin{array}{c}\text { Mass after } \\
\text { extraction(g) }\end{array}$} & \multirow{2}{*}{$\begin{array}{c}\text { Degree of } \\
\text { crosslinki } \\
\text { ng }(\%)\end{array}$} \\
\hline & $\begin{array}{l}\text { Filter } \\
\text { paper }\end{array}$ & $\begin{array}{c}\text { Microsp } \\
\text { heres }\end{array}$ & & \\
\hline 1.0 & 0.314 & 0.250 & 0.180 & 72.0 \\
\hline 1.5 & 0.309 & 0.272 & 0.199 & 73.1 \\
\hline 2.0 & 0.314 & 0.242 & 0.182 & 75.2 \\
\hline 2.5 & 0.314 & 0.250 & 0.192 & 76.8 \\
\hline 3.0 & 0.310 & 0.282 & 0.224 & 79.4 \\
\hline 3.5 & 0.309 & 0.250 & 0.195 & 78.0 \\
\hline 4.0 & 0.315 & 0.235 & 0.171 & 72.7 \\
\hline
\end{tabular}

It can known from the Tab. 1, with the increasing dosage of glutaraldehyde, the degree of crosslinking presents the trend of rise at first, but the trend reverse beyond a certain limit. When the dosage of glutaraldehyde is $3 \mathrm{~mL}$, its crosslinking degree can reaches $79.4 \%$, but when the dosage exceeds it, the crosslinking degree will not rise as before. The reason is that the reaction of aldehyde group and amino group has been finished.

\section{Analysis of encapsulation efficiency}

In the process of prepare betulinic acid/CS microspheres, many factors can have influence on the encapsulation efficiency, as a result, the practical application would be influenced. In this paper, the molecular weight of CS is a million, the ratio of betulinic acid to $\mathrm{CS}$ is $1: 4$, the dosage of Span- 80 is $3 \mathrm{~mL}$ and the glutaraldehyde is $2 \mathrm{~mL}$, the calculation of encapsulation efficiency is as following:

$$
\text { Drug-loading rate }=\frac{W_{c}}{W m} \times 100 \%
$$

In the formula above, $\mathrm{Wc}$ is the mass of betulinic acid that has existed in the microspheres, g. Wm is the mass of betulinic acid/CS microspheres[4-6]. The mass of microspheres used here is $0.03 \mathrm{~g}$, so the drug-loading of microspheres is $8.3 \%$.

In order to increase the drug-loading, the best process conditions was determined by orthogonal test as following: 
Tab.2 Orthogonal analysis of chitosan microspheres

\begin{tabular}{|c|c|c|c|c|c|c|}
\hline NO & $\begin{array}{c}\text { Mass of } \\
\text { microspheres( } \\
\mathbf{m g})\end{array}$ & $\begin{array}{c}\text { Mass of } \\
\text { emulisifier } \\
\text { (mL) }\end{array}$ & $\begin{array}{c}\text { Molecular } \\
\text { weigh of CS } \\
\text { (ten } \\
\text { thousands) }\end{array}$ & $\begin{array}{c}\text { m(betulinin } \\
\text { acid)/m(CS) }\end{array}$ & $\begin{array}{c}\text { Dosage of } \\
\text { glutaraldehyde } \\
\text { (mL) }\end{array}$ & $\begin{array}{c}\text { Drug-loadi } \\
\text { ng (\%) }\end{array}$ \\
\hline 1 & 30 & 1 & 20 & $1: 2$ & 2 & 6.9 \\
\hline 2 & 30 & 1 & 50 & $1: 4$ & 4 & 7.8 \\
\hline 3 & 30 & 1 & 100 & $1: 8$ & 4 & 9.3 \\
\hline 4 & 30 & 2 & 50 & $1: 4$ & 3 & 5.9 \\
\hline 5 & 30 & 2 & 100 & $1: 2$ & 3 & 4.3 \\
\hline 6 & 30 & 3 & 20 & $1: 8$ & 4 & 7.6 \\
\hline 7 & 30 & 3 & 50 & $1: 2$ & 2 & 8.9 \\
\hline 9
\end{tabular}

It can be known from the orthogonal test that, all of the dosage of emulsifiers and crosslinking, the ratio of betulinic acid to $\mathrm{CS}$ and the molecular weight of CS have influence on the drug-loading[7]. The effect of molecular weigh is the minimal and the ratio is the maximal. When the molecular weigh of CS is one million, the dosage of Span- 80 is $1 \mathrm{~mL}$ and glutaraldehyde is $4 \mathrm{~mL}$, the drug-loading can reach its maximum of $9.3 \%$.

\section{Analysis of sustained release}

$0.30 \mathrm{~g}$ of betulinic acid/CS microspheres was taken out and dissolved in hydrochloric acid solution at the concentration of $0.1 \mathrm{~mol} / \mathrm{L}$, constantly stirring for a while, put the mixture in a constant-volume of $50 \mathrm{~mL}$. Take out $2 \mathrm{~mL}$ of the mixture with pipette at $1 \mathrm{~h} 、 2 \mathrm{~h} 、 4 \mathrm{~h} 、 6 \mathrm{~h}$ 、 $8 \mathrm{~h} 、 10 \mathrm{~h} 、 12 \mathrm{~h} 、 16 \mathrm{~h} 、 20 \mathrm{~h}$ and $24 \mathrm{~h}$, respectively, the corresponding volume of hydrochloric acid solution is added at the same time. Determined the absorbance of the mixture with UV-VISIBLE spectrophotometer at the wavelength of $224 \mathrm{~nm}$, then the cumulative release rate of betulinic acid can be calculated with following formula:

$$
E_{r}=\frac{V_{e} \sum_{i}^{n-1} c_{i}+V_{\mathrm{o}} c_{n}}{m_{1} D}
$$

In the formula above, V0, is the initial release of concentration, $\mathrm{Ve}$ is displaced volume of release medium, $\mathrm{ci}$ is the concentration of drug at the ih time, $\mathrm{n}$ is the time of displacement, $\mathrm{ml}$ is the mass of microspheres, $\mathrm{D}$ is the drug-loading of microspheres[8,9]. 24h later, we can obtain the result of slow-release effect as following:
Tab.3 The relation between the cumulative release rate and time

\begin{tabular}{|c|c|c|}
\hline $\begin{array}{c}\text { Release } \\
\text { time/h }\end{array}$ & $\begin{array}{c}\text { Concentration } \\
\text { of betulinic } \\
\text { acid } / \mathbf{g} / \mathbf{L}\end{array}$ & $\begin{array}{c}\text { Cumulative release } \\
\text { rate } / \%\end{array}$ \\
\hline 1 & 0.010405 & 20.8 \\
\hline 2 & 0.020335 & 40.7 \\
\hline 4 & 0.030054 & 60.1 \\
\hline 6 & 0.037549 & 75.1 \\
\hline 8 & 0.040256 & 80.5 \\
\hline 10 & 0.043564 & 87.1 \\
\hline 12 & 0.046026 & 92.1 \\
\hline 16 & 0.048285 & 96.6 \\
\hline 20 & 0.049520 & 99.0 \\
\hline 24 & 0.049950 & 99.9 \\
\hline
\end{tabular}

It can be known from the Tab. 3 that, at the beginning of sustained-release, the betulinic acid in the microspheres was released at a high rate, the concentration of betulinic acid in solution increased rapidly, 4h later more than half of the drug has been released. When the microspheres released for about $16 \mathrm{~h}$, its cumulative release rate has passed $96 \%$, so it can be affirmed that the drug has been released absolutely. 


\section{Conclusion}

In the process of prepare the CS microspheres, the emulisifiers used here is Span-80, and the surface of the microspheres indicates that when the dosage of Span- 80 is $1 \mathrm{~mL}$, the particle size uniformity is the best, it ranged from $30 \mu \mathrm{m}$ to $180 \mu \mathrm{m}$.

Many factors could have an influence on drug-loading and encapsulation efficiency, and their order is the ratio of betulinic acid to CS is the maximal, the dosage of emulsifier and crosslinker take the second place, the molecular weigh of CS is the minimal.

Through the orthogonal test the optimum technological condition can be determined: the molecular weight of CS is one million, the dosage of Span- 80 is $1 \mathrm{~mL}$ and glutaraldehyde is $4 \mathrm{~mL}$, the reactive temperature is $60^{\circ} \mathrm{C}$, the stirring rate is $300 \mathrm{r} / \mathrm{min}$. At this time, the maximum of drug-loading is $9.3 \%$ and the maximal encapsulation is $79.4 \%$.

\section{Acknowledgement}

In this paper, the research was sponsored by the University Key Laboratory of Fine Chemical Industry of Heilongjiang Province from Qiqihaer University.

\section{References}

1. M. A. Berthol, S. K. Cremer, S. D. Kreuter. Control Rel, 39, 17-25 (2006).

2. Y. Xing. The Application of Chitosan in Drug Control-released . J. 26, 62-64 (2005).

3. T. D. Jiang, Chitosan, M, Chemical Industry Press, 1-4, (2007).

4. F. Li, M. Liu, K. Yao. Biomaterials. J. 23, 343-347(2012).

5. H. Shi. Biomaterials, J, 23, 4469-4471(2002).

6. C. Berkland, M. Kipper. Journal of Controlled Release. J. 94, 129-141(2012).

7. P.G.Srinivasa, J.A.Ramseh, C.S.Kuma. Journal of Food Engineering. J. 63, 79-85(2004).

8. M. L. Liu, S.D. Javalgill. Adv Drug Deliver. J. 51, 81-96(2001).

9. X. B. Xiong, J.M. Li. Sustained-Release Pellets. J. 12:705-720(2001). 